\title{
Micromachining of Diffractive Optical Elements Embedded in Bulk Fused Silica by Nanosecond Pulses
}

\author{
Francisco Javier Salgado-Remacha, Luis Miguel Sanchez-Brea, and Eusebio Bernabeu, Member, OSA
}

\begin{abstract}
Micro-optical devices embedded in transparent materials are usually manufactured focusing a pulsed laser in bulk fused silica. Under this condition, pulsewidth becomes the most important parameter that rules the size of the inscriptions. Ultrafast pulses (pico- and femtosecond pulses) avoid thermal effects and the results present a high efficiency. Nevertheless, nanosecond lasers are more available due the reduced costs. Therefore, a study of the optical behavior of embedded elements micromachined by nanosecond pulses is required. In this study, we show that this regime of pulses can still be used for engraving diffractive optical elements in transparent materials, regardless of the thermal damage. A Fresnel zone plate and a far-field beam shaper have been manufactured as an example of the functionality of these devices.
\end{abstract}

Index Terms-Diffraction, glass, integrated optics, laser application, micromachining, optical device fabrication, optical memories.

\section{INTRODUCTION}

A $\mathrm{S}$ a result of their promising prospect, micro- and nanooptics have experienced an increasing development in recent years. Micro-optical elements are not only important for scientific interest, but also they are successfully used in several applications, such as optical metrology, adaptive optics, or optical trapping [1], [2]. Several methods are available for the micromachining of these devices. Usually, diffractive optical elements (DOEs) are manufactured by photolithographic techniques [3].

DOEs are the key components for laser optics, sensor optics, and lighting applications. DOEs are used to diffract and shape light into the area of interest and they have the advantage to combine several optical functions needed for miniaturizing of optics. Their manufacture normally requires several processes, such us mask illumination, developing, and etching. There is always a clear interest on the minimization of the production costs [4]. Also, laser ablation with pulsed lasers has become important in the manufacturing of micro-optical applications. Specifically, laser micromachining of transparent dielectrics repre-

Manuscript received September 07, 2010; revised December 20, 2010; accepted December 30, 2010. Date of publication January 10, 2011; date of current version March 04, 2011. This work was supported by the Ministerio de Ciencia e Innovación under Project DPI2008-02391. The work of F. J. Salgado-Remacha was supported by the Ministerio de Ciencia e Innovación.

F. J. Salgado-Remacha, L. M. Sanchez-Brea, and E. Bernabeu are with the Universidad Complutense de Madrid, 28040 Madrid, Spain (e-mail: fjsalgado@fis.ucm.es; sanchezbrea@ fis.ucm.es; ebernabeu@ fis.ucm.es).

Color versions of one or more of the figures in this paper are available online at http://ieeexplore.ieee.org.

Digital Object Identifier 10.1109/JLT.2011.2104939 sents a good alternative for fabrication of waveguides [5], optical memories [6], or photonic crystals [7]. Although it is possible to engrave on the surface of the transparent sample [8], advantages of engrave in bulk transparent materials are clear. For example, it allows isolating the optical element from external agents [9], and also it is possible to fabricate 3-D elements [10]-[12].

Ultrafast laser inscription is used in a wide variety of glasses to manufacture embedded DOEs [13]. This technology requires a relative complex system. Several processes during the interaction light--matter with pulsed lasers are involved [14]. Thermal effects produced during the interaction between light and matter can produce undesired effects, which can be minimized using short- and ultrashort-pulses lasers (picosecondand femtosecond-pulses laser) [15]. In the range of long pulsewidths (nanosecond), microcracks appear surrounding the damaged zones, even at fluencies near the laser-induced damage threshold of the material [10]. For that reason, the efforts in the micromachining of transparent materials are focused in the short- and ultrashort-pulses laser. In this study, we use a simple nanosecond laser to engrave DOEs in bulk fused silica. Although this technique produces elements with lower efficiency than photolithography or femtosecond laser inscription, it can be performed in just one single step. Then, it can be useful for rapid prototyping of DOEs. Actually, most of the studies in this field are centered in femtosecond laser micromachining. On the contrary, we prefer to show, with this study, the optical behavior of DOEs in transparent materials micromachined by means of nanosecond-pulses trains. Technical works concerning to bulk fused silica micromachining make incidence, especially in the use of ultrashort-pulse lasers. The low number of works in this field using nanosecond-pulse lasers are usually centered in the study of the light--matter interaction. In fact, the phenomenology involved in this kind of interaction is not yet well known due the amount of effects implicated during the process. The use of DOEs micromachined by this kind of lasers is not well studied. In this sense, this study pretends to illustrate the possibilities of nanosecond-pulse lasers in the field of bulk fused silica micromachining.

In Section II, we analyze how binary-amplitude DOEs can be fabricated in bulk fused silica with a nanosecond laser, performing an optimization of the parameters involved. In Section III, we show two examples of binary-amplitude DOEs that has been fabricated in bulk fused silica, and we also show the optical results obtained with them. In particular, we have engraved a Fresnel zone plate (FZP) and a beam shaper (BS) 
that generates a certain intensity distribution in the far field. Finally, conclusions are given in Section IV.

\section{Micromachining OF FuSED SiLICA By NANOSECOND PULSES ABLATION}

For the fabrication of DOEs in bulk fused silica, we have used a $\mathrm{Nd}: \mathrm{YVO}_{4} Q$-switched laser (navigator I from Spectra Physics). The nominal working wavelength of this laser head is $\lambda=1064 \mathrm{~nm}$. Using a third-harmonic generator (THG), we obtain laser peaks at $\lambda=355 \mathrm{~nm}$. Fused silica is not transparent for this wavelength, and the energy is absorbed by the material. The beam waist of the focal spot for the Gaussian beam with radius $r$ produced by a lens with focal $f$ is as follows:

$$
\omega_{0}^{G}=\frac{\lambda}{\pi}\left(\frac{f}{r}\right) M^{2}
$$

where $f=46 \mathrm{~mm}$ and $r=4.5 \mathrm{~mm}$ (using a beam expander $\times 15$ before the final focusing lens). The nominal beam quality factor is $M^{2}=1.3$. Under this configuration, the beam waist is $\omega_{0}^{G}=2.99 \mu \mathrm{m}$. Then, the spot area results in $A_{\text {focus }}=\pi\left(\omega_{0}^{G}\right)^{2}=7.05 \mu \mathrm{m}^{2}$.

With the THG, the maximum peak power $P$ is obtained at $20-\mathrm{KHz}$ pulse repetition rate, with $P=2 \mathrm{~W}$, and the pulse energy $E_{\text {pulse }}$ is $E_{\text {pulse }}=2 \mathrm{~W} / 20 \mathrm{KHz}=0.1 \mathrm{~mJ}$. Then, the maximum fluence attainable at the focus is

$$
F_{\text {focus }}^{\mathrm{Max}}=\frac{E_{\text {pulse }}}{A_{\text {focus }}}=1.4 \times 10^{3} \mathrm{~J} / \mathrm{cm}^{2} .
$$

The transparent sample is located over a two axis linear stage. The laser beam is focused in bulk glass. The system is arranged to work as a plotter; therefore, we can manufacture the masks pixel by pixel.

As we have mentioned, thermal effects become important in the long-pulses regime due to the strong dependency between thermal and mechanical properties of the sample.

For this study, we use fused silica microscope slides, since they are inexpensive and easy to find. The thickness of the samples is $1 \mathrm{~mm}$ and they present a high-quality surface. We have used a repetition frequency of $20 \mathrm{KHz}$, with a pulsewidth of $\tau=12 \mathrm{~ns}$. With this configuration, we analyze the behavior of the glass for multipulse damage. In order to minimize the thermal damage and energy propagation, we use pulses with low energy. The shape of the obtained damages are shown in Fig. 1 . The evolution of the damages with the number of pulses and the fluence can be appreciated in Fig. 1(b), and a more detailed image is shown in Fig. 1(b). As it can be observed, there are two different regions of damage. One of them consists of dark zones, appearing due the accumulation of pulses. The nature of these zones is usually assumed as process of heating/melting in the glass with a transition dielectric/metal [16]. After a certain number of pulses, appear highly absorbing zones with a metallike state inside the dielectric. Our purpose is to use these highly absorbing zones as dark zones for binary-amplitude masks. The other kind of damage consists of breaks in the glass due the high temperatures reached with the focalized laser that can induce some kind of aberrations in the wavefront.

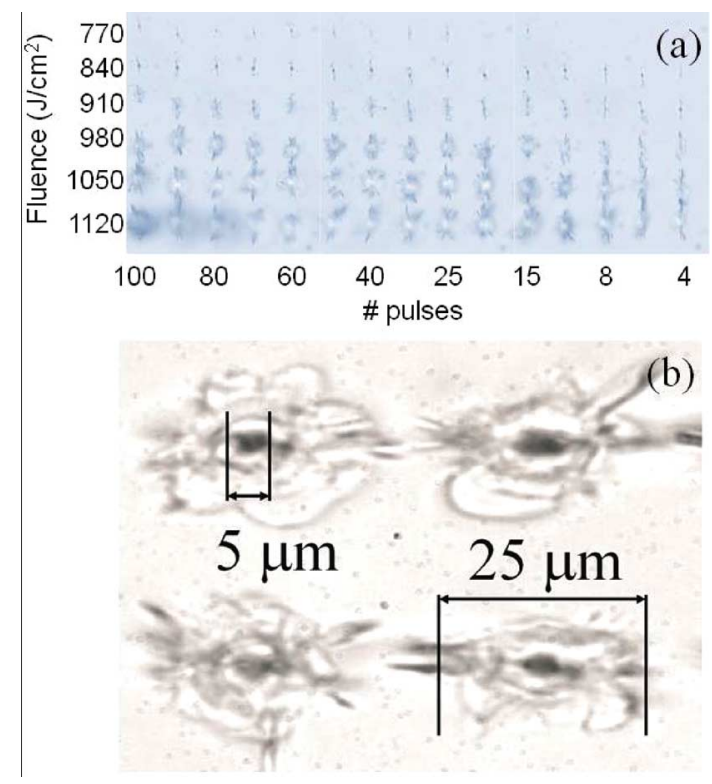

Fig. 1. Calibration process. (a) Calibration of damages for different values of fluence and different number of pulses. (b) Shape of the damages obtained with $\tau=12 \mathrm{~ns}$ and $F=1.4 \times 10^{3} \mathrm{~J} / \mathrm{cm}^{2}$. ( $\times 20$ optical microscopy.) Dark zones are surrounded by thermal break zones.

The widths of the dark zones, along $x$ - and $y$-axes, obtained for different number of pulses and different fluences, are collected in Fig. 2(a) and (b) . These data were obtained after averaging over 256 measurements for each value of fluence and for each number of pixels. We can appreciate that there exist a clear dependence between the width of the dark zone and the fluence at focus. The dependence with the number of pulses, however, is not so clear. Moreover, there exist a strong asymmetry between $x$ axis and $y$-axis. In order to reach a good accuracy, it is important to obtain a circularlike pixel shape. In Fig. 2(c), the difference between dark zones width along $x$ - and $y$-axes is plotted. The minimum difference (and, consequently, the more circularlike shape) is obtained for low fluencies.

Moreover, we have experimentally found that when the thermal damages around two dark zones are too close, breaks can propagate along the glass, thus destroying it. The evolution of the width of the thermal damages is shown in Fig. 3. In addition to the effect of the fluence, a dependence with the number of pulses can be observed. Moreover, for a low number of pulses, the shape and width of this thermal damage are not constant. Repeatability is an important factor in diffractive elements fabrication. For these reasons, our interest is to find an optimal solution, where a number of pulses produce the smaller thermal damage width and with a constant shape of the dark zones. We have performed a calibration with different power, number of pulses and separation of dark zones, finding that the optimal solution without breaking the glass results for 20 pulses of $56 \mu \mathrm{J}$, with a fluence in focus of $36 \mathrm{~J} / \mathrm{cm}^{2}$ and a separation of $10 \mu \mathrm{m}$ between pixels. The lack of works with DOEs micromachined by nanosecond-pulse lasers is probably due to the impossibility to avoid the microcraks due the thermal effects, as it is shown in the calibration data of Figs. 2 and 3. The chosen parameters minimize thermal damage and anisotropy of the dark zones, allowing also a good ratio of repeatability. 
(a)

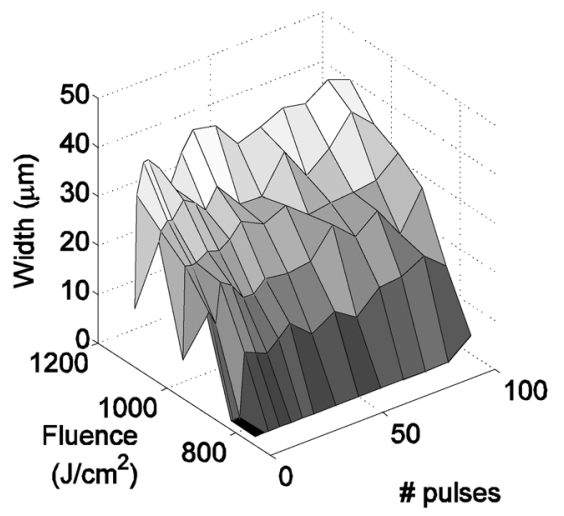

(b)

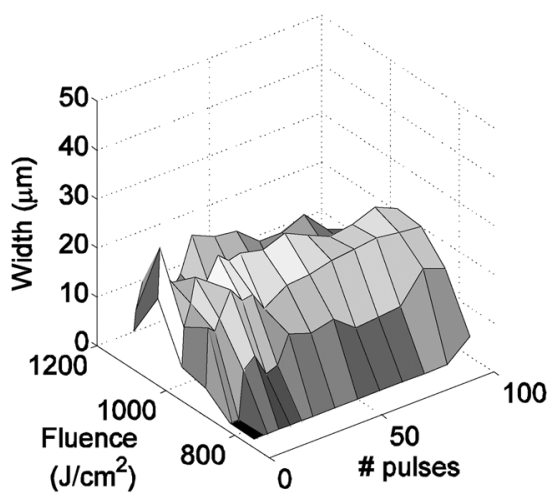

(c)

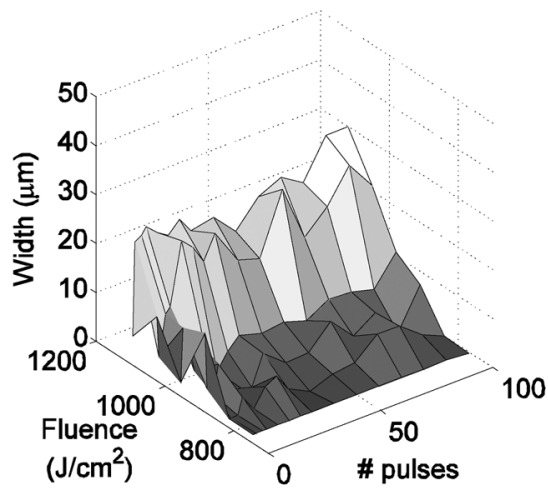

Fig. 2. Dark zones widths at $20 \mathrm{KHz}$ and $\tau=12 \mathrm{~ns}$ for different number of pulses and different values of fluence. (a) Along $x$-axis. (b) Along $y$-axis. (c) Differences between width along $x$ axis and width along $y$-axis.

\section{BINARY-AMPLITUDE DOES}

Once we have analyzed how laser engraving affects the pixels generation in bulk glass, now we can fabricate DOEs. Due to the characteristics of the laser damage in bulk fused silica, we can engrave binary DOEs that modify the amplitude of the incident field. The engraved DOE consists of a pixelated mask with two levels of transmitivity: opaque and transparent pixels. In Fig. 4(a), schematic view of the system is shown. In order to study the optical behavior, we have engraved two DOEs with different characteristics: a FZP and a DOE for beam shaping, generating a certain intensity distribution in the far field. (a)

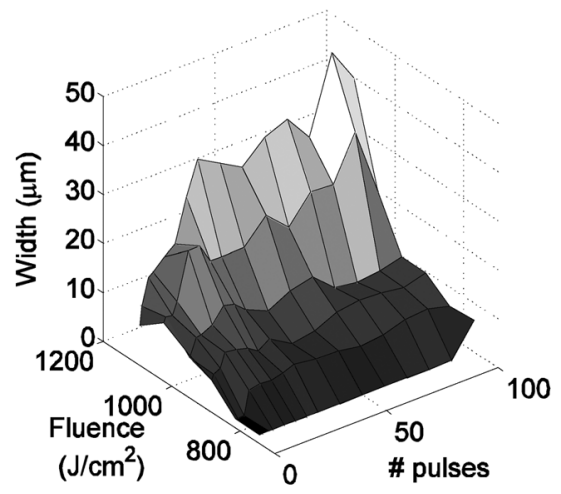

(b)

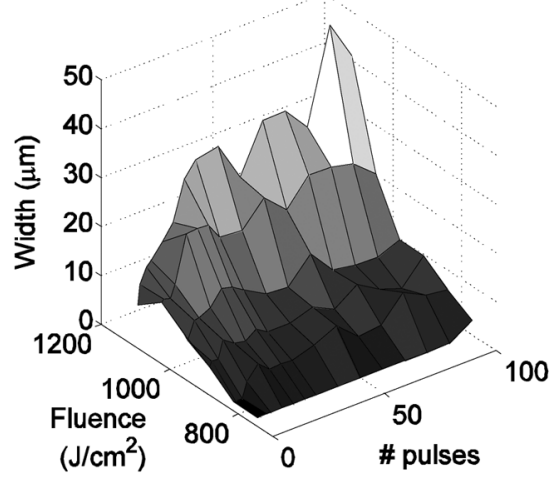

(c)

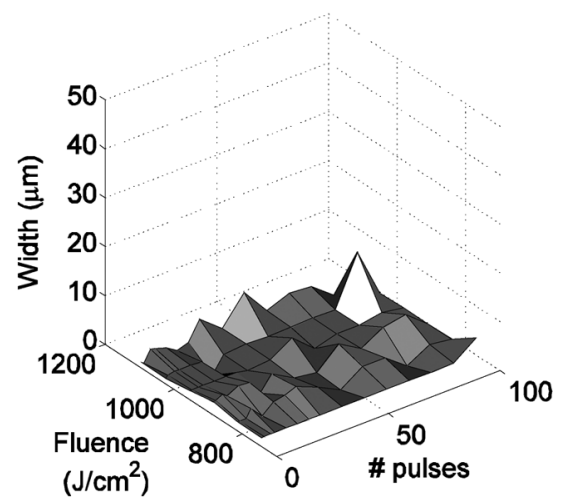

Fig. 3. Thermal breaks widths at $20 \mathrm{KHz}$ and $\tau=12 \mathrm{~ns}$ for different number of pulses and different values of fluence (a) along $x$ axis and (b) along $y$-axis. (c) Difference between width along $x$ axis and width along $y$-axis.

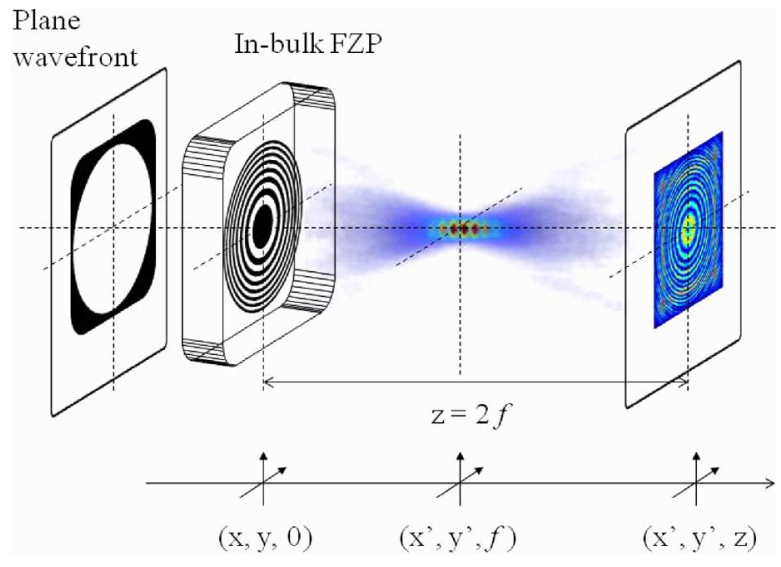

Fig. 4. Schematic view of the system working with an embedded FZP. 


\section{A. Fresnel Zone Plates}

An FZP is one of the most used micro-optical elements, since they concentrate light at the focal point placed at a distance $f$ from the FZP. These diffractive lenses present some advantages over conventional lenses. They can be used, for example, over spectral regions, where refractive lenses are useless (as UV), or in applications, where low weight is required. FZP consists of alternate opaque and transparent rings. The transmitivity function $L(x, y)$ results in the following [17]:

$$
L(x, y)= \begin{cases}1, & \text { if } \sqrt{m \lambda f} \leq x^{2}+y^{2}<\sqrt{(m+1) \lambda f} \\ 0, & \text { if } \sqrt{(m-1) \lambda f} \leq x^{2}+y^{2}<\sqrt{m \lambda f}\end{cases}
$$

where $\lambda$ is the wavelength, $f$ is the focal distance from the FZP, and $m$ is an integer $m=1,2,3, \ldots$ In Fig. 5(a), we show an example of a FZP for a focal length $f=450 \mathrm{~mm}$. Also, a microscopic view of a FZP engraved into a bulk fused silica sample is shown in Fig. 5(b).

FZP has been designed to work in transmission at $\lambda=632.8$ nm. Following the schemes of Fig. 4, and illuminating with a laser diode emitting at $\lambda=632.8 \mathrm{~nm}$, modulated light after the DOEs is collected with a CMOS (UI-1220-M by U-Eye, Germany), with a pixel pitch of $6 \mu \mathrm{m}$. The camera is mounted over a motorized linear stage; therefore, it can travel along propagation axis and images are acquired at any desired plane. Under these conditions, we have obtained the intensity distribution along the propagation axis [see Fig. 5(c)]. This propagation is compared with a numerical simulation. We have used the scalar theory for the light propagation, in particular the Rayleigh--Sommerfeld approach, using a fast-Fourier-transform-based direct integration method [18]. With this algorithm, we have computed the propagation around the focus of the binary Fresnel lens, assuming thin element approximation [see Fig. 5(d) ]. The results are very similar in both cases except for a slight asymmetry in the experimental intensity distribution.

\section{B. Diffractive BS (DBS) in the Far Field}

Another kind of diffractive element that can be engraved in bulk fused silica is a BS, which is an optical element that transforms an incoming wavefront $U(x, y, 0)$ into a desired intensity distribution at an observation plane $z$, as it is shown in Fig. 6. When the wavefront is modulated by means of diffraction, it is called diffractive BS (DBS) [1].

In order to design the DBS, we have considered Fraunhoffer approach. We have used the iterative Fourier transform algorithm (IFTA), thus adding the restriction of binary and amplitude elements [19]. The BS has been designed to work in transmission at $\lambda=632.8 \mathrm{~nm}$. As an example, in Fig. 7(a) and (b), we can find the desired far-field intensity distribution and the binary DBS designed with the IFTA algorithm. Since the DBS is binary, we always obtain at the observation plane, the desired intensity distribution and also the symmetrical image of this field, according to the mathematical properties of the Fourier transform. The Fourier transform of Fig. 7(b) is shown in Fig. 7(c), where we can observe the double image.

In Fig. 8(a), we can see an optical microscopy image of the manufactured DBS [designed in Fig. 7(b)] and also the intensity distribution in the far field obtained with this DOE in Fig. 8(b). (a)

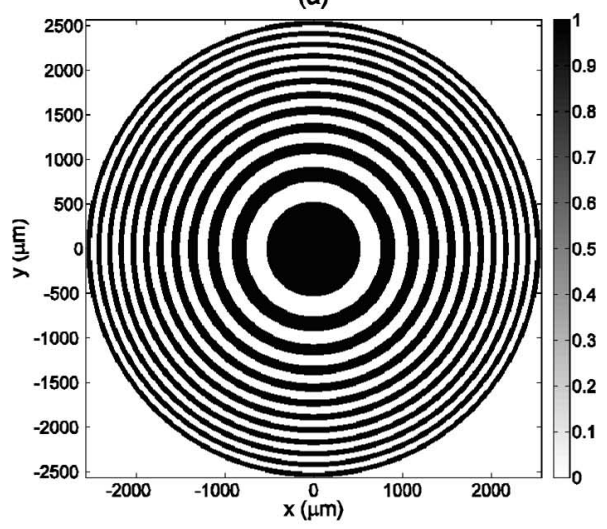

(b)

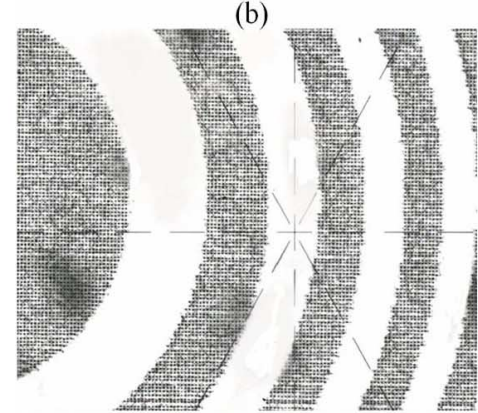

(c)

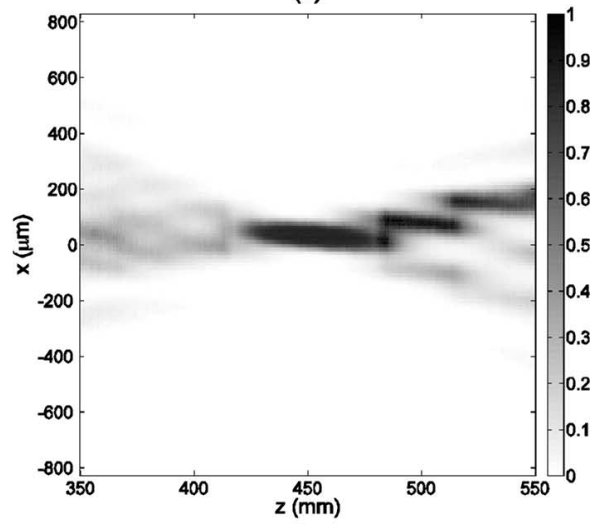

(d)

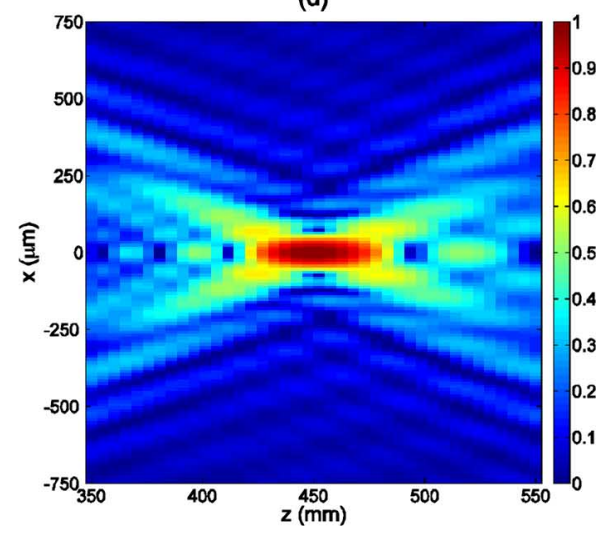

Fig. 5. (a) Binary-amplitude FZP with $f=450 \mathrm{~mm}$. (b) Microscopic image of the FZP in bulk fused silica ( $\times 5$ optical microscopy). Separation between pixels is $10 \mu \mathrm{m}$. (c) Experimental propagation after the embedded FZP around the principal focus. (d) Simulation obtained with Rayleigh--Sommerfeld approach for the same parameters.

As we can see, the experimental results are very similar to the expected [see Fig. 7(c)]. 


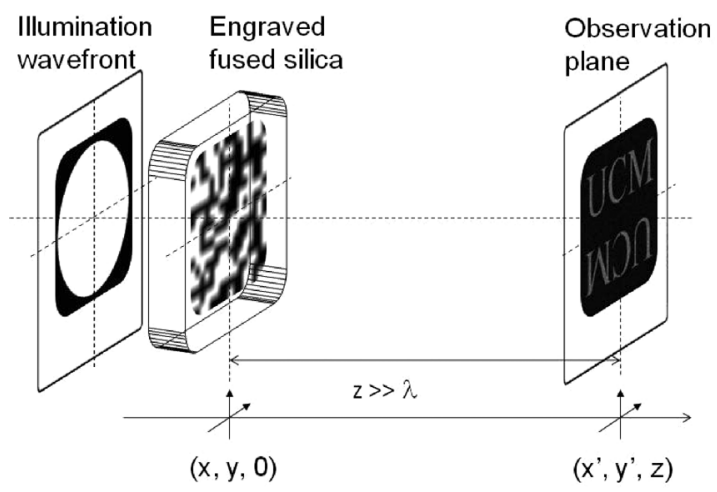

Fig. 6. View of the system working with an embedded DBS.

(a)

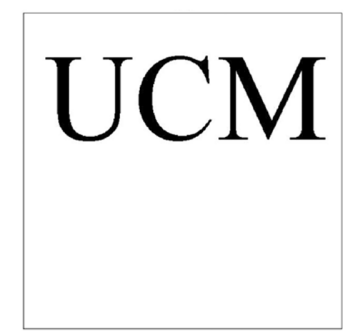

(b)

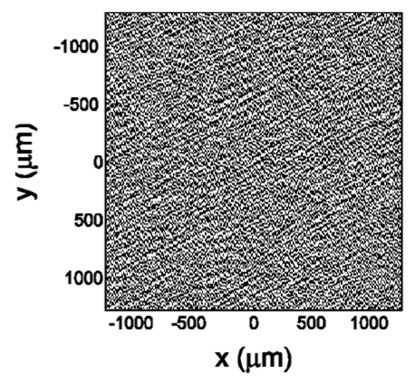

(c)

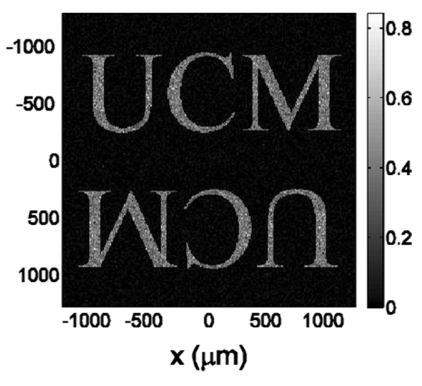

Fig. 7. (a) Desired intensity distribution at the far field. (b) Binary-amplitude DBS obtained with IFTA designed to obtain the intensity distribution given in (a). (c) Fourier transform of the DBS (central maximum peak was removed).

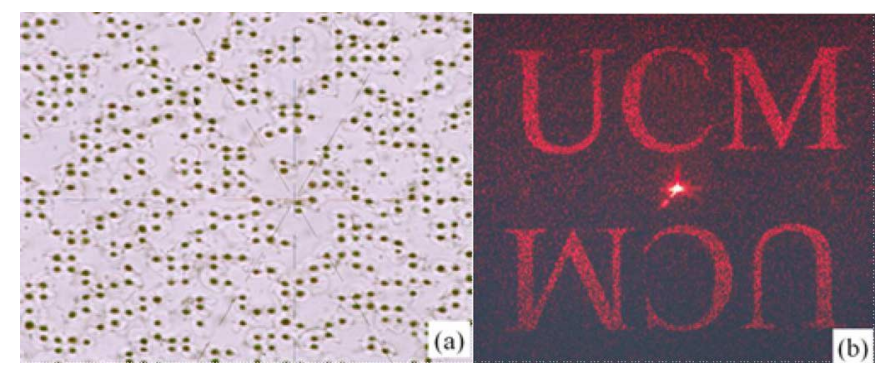

Fig. 8. (a) Microscopic image of the DBS in bulk fused silica $(\times 20$ optical microscopy). Separation between pixels is $10 \mu \mathrm{m}$. (b) Experimental propagation after the embedded DBS in the far field. The results are very similar to the expected in Fig. 7(c).

In order to numerically evaluate the behavior of the DBS, we can follow the method explained in [4]. We choose a region in the objective field in which we define a window surrounding the signal area (the pixel in which the intensity should be maximum). We considered this window as signal intensity $i_{\text {signal }}$. The rest of the region is considered as noise intensity $i_{\text {noise }}$. We also measured the standard deviation $\sigma$ in both areas. This

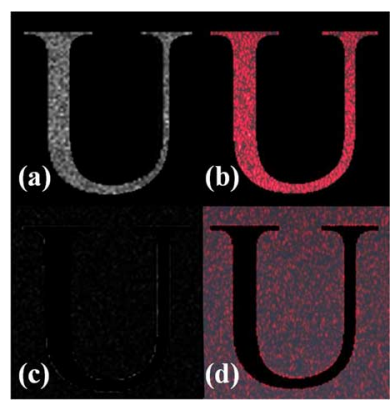

Fig. 9. (a) Signal window for the simulated propagation. (b) Signal window for the experimental propagation. (c) Noise window for the simulated propagation. (d) Noise window for the experimental propagation.

TABLE I

Numerical EVAluation of the Simulated Propagation AND the EXPERIMENTAL PROPAGATION

\begin{tabular}{|c|c|c|c|}
\hline & $i_{\text {signal }} / i_{\text {noise }}$ & $\sigma_{\text {signal }}$ & $\sigma_{\text {noise }}$ \\
\hline designed & 21.15 & 0.136 & 0.018 \\
\hline experimental & 7.37 & 0.262 & 0.153 \\
& & & \\
\hline
\end{tabular}

process is applied to the experimental propagation and also to a simulated propagation of the designed element in Fig. 7(a).

In Fig. 9, we show the windows used for the numerical evaluation. We collected all the values in Table I. The values of the intensity are normalized to the area of the signal window.

As we can appreciate, the SNR for the experimental propagation is three times higher than the SNR of the designed DOE. The loss of SNR is due the high level of noise, attached to the imperfections in the DBS. In the same way, the standard deviations of both signals are comparable, but the standard deviation of the noise is much higher for the experimental propagation. In this sense, we considered that the embedded DOEs fabricated are useless for applications in which the loss of energy by fabrication errors is not so important as to obtain a desirable distribution field with a low cost, with the value added for the protection of the diffractive element by the substrate.

\section{CONCLUSION}

We have analyzed the fabrication of the embedded DOEs by means of nanosecond pulses. Since the availability of this kind of lasers, this technique represents a good alternative to ultrashort-pulses laser processing. The accumulation of pulses over a region in bulk glass produces dark zones, surrounded by thermal breaks due to the long pulses regime. Then, the fabricated DOE modulates light amplitude in two levels (binary-amplitude elements). Due the length of the pulsewidth used, there appear break zones around the irradiated areas.

Although these broken zones should produce a loss of efficiency, this technique is much fast and simpler than standard photolithographic process, and inexpensive in comparison with the ultrashort-pulses micromachining. In order to illustrate the behavior of this kind of DOEs, a FZP and a far-field BS have been manufactured in bulk glass, comparing the results with numerical simulations. An analysis of these devices shows that although they present some elevated level of noise, they are able to reconstruct successfully the objective pattern, with a good 
quality. For this reason, this technique can be useful for rapid prototyping of in-bulk DOEs.

\section{REFERENCES}

[1] J. Turunen and F. Wyrowski, Diffractive Optics for Industrial and Commercial Applications, J. Turunen and F. Wyrowski, Eds. Berlin, Germany: Akademie-Verlag, 1997.

[2] H. P. Herzig, Micro-Optics. Elements, Systems and Applications, H. P. Herzig, Ed. London, U.K.: Taylor \& Francis, 1997.

[3] T. J. Suleski, "Fabrication trends for free-space microoptics," J. Lightw. Technol., vol. 23, no. 2, pp. 633-646, Feb. 2005.

[4] I. Moreno, A. Martínez-García, L. Nieradko, J. Albero, and C. Gorecki, "Low cost production of computer-generated holograms: From design to optical evaluation," J. Eur. Opt. Soc. Rap. Public., vol. 5, no. 10011, pp. 1-9, Apr. 2010.

[5] C. B. Schaffer, A. Brodeur, J. F. García, and E. Mazur, "Micromachining bulk glass by use of femtosecond laser pulses with nanojoule energy," Opt. Lett., vol. 26, pp. 93-95, Jan. 2001.

[6] S. Juodkazis, A. V. Rode, E. G. Gamaly, S. Matsuo, and H. Misawa, "Recording and reading of three-dimensional optical memory in glasses," Appl. Phys. B, vol. 77, pp. 361-368, Aug. 2003.

[7] V. Mizeikis, K. K. Seet, S. Juodkazis, and H. Misawa, "Three-dimensional woodpile photonic crystal templates for the infrared spectral range ," Opt. Lett., vol. 29, pp. 2061-2063, Sep. 2004.

[8] B. Hopp, T. Smausz, and M. Bereznat, "Processing of transparent materials using visible nanosecond laser pulses," App. Phys. A, vol. 87, pp. 77-79, Jan. 2007.

[9] W. Watanabe, D. Kuroda, and K. Itoh, "Fabrication of Fresnel zone plate embedded in silica glass by femtosecond laser pulses ," Opt. Exp., vol. 10, pp. 978-983, Sep. 2002.

[10] E. N. Glezer, M. Milosavljevic, L. Huang, R. J. Finlay, T. H. Her, J. P. Callan, and E. Mazur, "Three-dimensional optical storage inside transparent materials," Opt. Lett., vol. 21, pp. 2023-2025, Dec. 1996.

[11] T. Tanaka and S. Kawata, "Comparison of recording densities in threedimensional optical storage systems: Multilayered bit recording versus angularly multiplexed holographic recording," J. Opt. Soc. Amer. A, vol. 13, pp. 935-943, May 1996.

[12] Y. Li, Y. Dou, R. An, H. Yang, and Q. Gong, "Permanent computer-generated holograms ebedded in silica glass by femtosecond laser pulses ," Opt. Exp., vol. 13, pp. 2433-2438, Apr. 2005.

[13] S.-H. Cho, W.-S. Chang, K.-R. Kim, and J.-W. Hong, "Femtosecond laser embedded grating micromachining of flexible PDMS plates," Opt. Commun., vol. 282, pp. 1317-1321, Apr. 2009

[14] E. G. Gamaly, A. V. Rode, and B. Luther-Davies, "Ablation of solids by femtosecond laser: Ablation mechanism and ablation thresholds for metals and dielectrics," Phys. Plasmas, vol. 9, pp. 949-957, Mar. 2002.
[15] P. Gibbon, Short Pulse Laser Interactions With Matter. London, U.K.: Imperial College Press, 2005.

[16] O. Efimov, S. Juodkazis, and H. Misawa, "Intrinsic single- and multiple-pulse laser-induced damage in silicate glasses in the femtosecond-to-nanosecond region," Phys. Rev. A, vol. 69, pp. 042903-1-042903-7, Apr. 2004.

[17] E. Hecht, "Fresnel diffraction," in Optics. Reading, MA: Addison Wesley, 2002, ch. 10, sec. sec. 3 .

[18] F. Shen and A. Wang, "Fast-Fourier-transform based numerical integration method for the Rayleigh-Sommerfeld diffraction formula," Appl. Opt., vol. 45, pp. 1102-1110, Feb. 2006.

[19] F. Wyrowski, "Iterative quantization of digital amplitude holograms,", App. Opt., vol. 28, pp. 3864-3870, Sep. 1989.

Francisco Javier Salgado-Remacha was born in Jaca, Spain, in 1980. He received the degree in physics from the Universidad de Zaragoza, Zaragoza, Spain, in 2005, and the M.Sc. degree from Universidad Computense de Madrid, Madrid, Spain, in 2008, where he is currently working toward the Ph.D. degree.

Luis Miguel Sanchez-Brea was born in Madrid, Spain, in 1972. He received the M.Sc. degree, and the Ph.D. degree in physics from the Universidad Complutense de Madrid, Madrid, Spain, in 1996 and 2001, respectively.

He was an Assistant Professor and a "Ramon y Cajal" Researcher at the Optics Department, Universidad Complutense de Madrid, where he is currently an Associate professor. He was also a R\&D Technician at Fagor Automation S Coop. He has authored around 40 papers. His research interests include applied optics metrology and image processing.

Eusebio Bernabeu was born in Logroño, Spain, in 1944. He received the M.Sc. degree, and the Ph.D. degree in 1969 in physics, both from the University of Zaragoza, Zaragoza, Aragon, Spain, in 1966 and 1969, respectively.

He was at l'Ecole Normale Superieur, at the Laboratoire de l'Horloge Atomique in Paris, at the International Center of Theoretical Physics of Trieste, and at the Universities of Zaragoza, Autónoma de Barcelona, and Complutense de Madrid. Since 1986, he has been a Professor at the Physics Faculty, Universidad Complutense de Madrid, Madrid, Spain. He has authored more than 250 papers and ten books. His current research interests include optoelectronics, optical sensors, fiber optical devices, and optical industrial inspection and metrology.

Prof. Bernabeu is a member of the Optical Society of America (OSA), the Society of Photo-Optical Instrumentation Engineers (SPIE), and European Physical Society (EPS). 